\title{
Concurrence chez Vicia sativa L et Avena sativa L. I. Effets des contraintes hydriques et nutritionnelles sur la morphogenèse et la démographie des organes
}

\author{
M Ouknider ${ }^{*}$, P Jacquard 1, D Elkhyari 2 \\ 1 CNRS, Centre Louis-Emberger, BP 5051, 34033 Montpellier, France; \\ 2 département de biologie, faculté des sciences, université Mohamed V, Rabat, Maroc
}

(Reçu le 27 février 1990; accepté le 25 mars 1991)

\begin{abstract}
Résumé - La variation des paramètres morphogénétiques et démographiques, chez la vesce et l'avoine, a été étudiée expérimentalement sous l'influence du régime hydrique, de la déficience potassique et du niveau d'azote. Dans les limites des contraintes imposées, les effets de la concurrence intraspécifique (densité) et de la concurrence interspécifique (association) ont été mis en évidence. Chez les 2 espèces, la concurrence intraspécifique a agi de la même façon en ralentissant le rythme d'émergence foliaire et en racourcissant la durée de vie des feuilles. Les effets sont d'autant plus marqués que l'intensité de la contrainte est élevée. Un régime hydrique contraignant augmente la longévité des feuilles. La déficience en potassium pour un niveau d'azote donné accroít le phyllochrone** de l'ordre de $20 \%$.
\end{abstract}

vesce / avoine / association / morphogenèse / concurrence

Summary - Competition in Vicia sativa L and Avena sativa L. I. Effects of water stress and nutrient availability on organ morphogenesis and demography. The variation of morphogenetic and demographic parameters for vetch and oat was experimentally studied under different water supply regimes, potassium deficiency and nitrogen levels. The results for oat show that the rate of emergence of the leaves on the main stem is dependent on leaf order (fig $1 a, b)$. On the primary tillers, the mean degree-day duration of a phyllochron depends on tiller order. Under the imposed constraints, the effects of intraspecific (density) and interspecific (association) competition were evidenced. Potassium deficiency increased the duration of the phyllochron for the 2 species (fig 2). The rate of leaf emergence presented a high interaction between the rank of the phyllochron and the levels of nitrogen and potassium. Intraspecific competition slowed down the rhythm of leaf emergence and reduced leaf longevity in both species (figs 5,6$)$. There was a strong effect of the water regime on vetch (fig 3). When potassium availability decreased, leaf emergence was slowed down. Increasing the intensity of the water constraints resulted in prolonged leaf longevity. There was a clear effect of potassium on the longevity of vetch leaves (fig 7). Potassium deficiency for a given nitrogen level increased the phyllochron by $20 \%$.

vetch / oat / mixture / morphogenesis / competition

\footnotetext{
*Adresse actuelle : ENA, département d'agronomie, BPS 40, Meknès, Maroc

** Phyllochrone : intervalle de temps qui sépare l'émergence de 2 feuilles successives.
} 


\section{INTRODUCTION}

La croissance d'une plante peut être décrite par des paramètres démographiques dont les relations avec la biomasse ont été étudiées par Bazzaz et Harper (1977) sur le lin, Maitre et al (1985) sur le trèfle violet. L'élément prépondérant est alors la vitesse intrinsèque de croissance de chaque module ou unité de tallage ou de ramification. Maitre et al (1985) ont montré que chaque module peut potentiellement accomplir un cycle biologique complet, de son émergence à sa sénescence; mais l'augmentation de la densité de peuplement, par exemple, se traduit par une diminution des nombres de ramifications et d'inflorescences (Donald, 1963) et de la vitesse d'apparition des feuilles (Turkington, 1983).

La biomasse élaborée par individu est la conséquence de la démographie des organes et des modifications plastiques induites par les interférences biologiques causées par la proximité des voisins (Harper, 1961). Mack et Harper (1977) ont montré que la variabilité du poids des individus est expliquée par l'action des voisins se trouvant à $2 \mathrm{~cm}$. Turkington (1983) indique que la réponse à l'interférence entre plantes voisines se manifeste par un taux de croissance réduit. Les plantes soumises à une contrainte de densité du peuplement présentent une distribution log-normale des biomasses individuelles, biaisée vers un excédent des valeurs faibles (Obeid et al, 1967; Ogden, 1970). Sur Bromus erectus, Jacquard (1984) a montré que la structure de la population de talles est fortement influencée par la densité. Des différences marquées des caractéristiques démographiques entre descendants ont été montrées par Fowler et Antonovics (1981).

Le présent travail constitue le prolongement d'études antérieures réalisées sur vesce-avoine (Ouknider et Jacquard, 1986, 1988) et se propose d'étudier l'organisation morphologique des 2 espèces, en culture pure et en association, dans différents environnements : densité de peuplement, régime hydrique et contraintes minérales.

\section{MATÉRIEL ET MÉTHODES}

Les résultats qui sont présentés s'appuient sur 3 dispositifs dont l'objectif n'était pas seulement une évaluation de la biomasse mais aussi une étude du mode d'élaboration de celle-ci : phyllochrones, rythme d'apparition des tiges et durée de vie des feuilles.

\section{Matériel végétal}

Le matériel végétal dans les expériences 1 et 2 était constitué d'une légumineuse : la vesce (Vicia sativa L), variété Septimane, et d'une graminée : l'avoine (Avena sativa L), variété Graine noire. Pour l'expérience 3 , on a utilisé un matériel local : vesce, variété 6242 , et avoine, variété 153 tutrice créée par l'INRA du Maroc.

\section{Dispositifs expérimentaux et facteurs étudiés}

\section{Expérience 1}

Elle testait l'effet de 4 densités : $4,8,16$ et 40 individus par pot, en culture pure et en association, correspondant respectivement à 100, 200,300 et 500 plantes $/ \mathrm{m}^{2}$. Le dispositif était placé à l'extérieur et irrigué par un système goutte à goutte.

\section{Expérience 2}

Cette expérience, conduite en hydroponie à des fins physiologiques, a été conçue pour l'étude de l'effet d'une carence minérale sur la variabilité des paramètres morphogénétiques et démographiques. Les solutions nutritives comportaient 3 concentrations de potassium notées : $\mathrm{K}_{6}=0,6 \mathrm{mN} ; \mathrm{K}_{3}=0,3 \mathrm{mN}$ et $\mathrm{K}_{1}=$ $0,1 \mathrm{mN}$, combinées à 2 niveaux d'azote $\left(\mathrm{N}_{14}=1,4 \mathrm{mN}\right.$ et $\mathrm{N}_{3}=0,3 \mathrm{mN}$ de $\mathrm{NO}_{3}{ }^{-}$). La solution la plus riche, $\mathrm{N}_{14} \mathrm{~K}_{6}$, est équilibrée, faiblement concentrée, et constitue la solution de référence. À chacune des solutions correspondraient 3 types de culture : la vesce pure, l'avoine pure et l'association. L'unité de culture comportait 8 plantes poussant en pot sur sable siliceux, à raison de 2 plantes par pot. L'irrigation, automatique, se déclenchait toutes les $2 \mathrm{~h}$ pendant $15 \mathrm{~min}$.

\section{Expérience 3}

Elle est conduite sous serre, la densité et le régime hydrique y ont été contrôlés. Quatre densités de peuplement, en culture pure et en association, ont été testées : 4, 8, 16 et 32 individus par pot, correspondant respectivement à $50,100,200$ et 400 individus $/ \mathrm{m}^{2}$. Pour l'alimentation en eau, 3 régimes ont été appliqués durant la période d'expérimentation : un régime considéré comme non limitant $\left(H_{1}\right)$, un régime à contrainte hydrique intermédiaire $\left(\mathrm{H}_{2}\right)$, et un régime à forte contrainte hydrique $\left(H_{3}\right)$. Pour le régime $H_{1}$, l'humidité du sol était maintenue proche de la capacité au champ (humidité pondérale de $30 \%$ ). Pour cela, les pots étaient pesés régulièrement, et à chaque fois que le poids enregistré correspondait à une humidité inférieure à $22 \%$, on apportait la quantité d'eau nécessaire pour obtenir à nouveau une humidité équivalente à la capacité au champ. 
L'expérience comportait 180 pots (4 densités $\times 3$ régimes hydriques $\times 3$ modes de culture, avec 5 répétjtions). Avant le repiquage des plantules, l'ensemble des pots a été ramené à une humidité correspondant au régime $\mathrm{H}_{2}$ d'irrigation. L'application des différents traitements a coïncidé avec l'apparition de la première feuille pour l'avoine et de la deuxième feuille pour la vesce.

\section{Observations et mesures}

Au cours du cycle cultural, les états de croissance et de développement ont été suivis sur la vesce et l'avoine.

\section{Mesures morphogénétiques non destructives}

Axées essentiellement sur le rythme d'apparition des feuilles et des tiges et sur la durée de vie des feuilles, elles ont porté sur 3 invididus par traitement. Les observations ont été faites quotidiennement depuis la levée jusqu'aux stades épiaison (avoine) ou floraison (vesce). Les plantes soumises au contrôle ainsi que les talles (graminées) et rameaux (légumineuses) ont été individualisés par des bagues. Sur les feuilles, un marquage des émergences a été adopté ce qui permet de reconnaître leur ordre. La méthode de marquage foliaire utilisée (fines perforations) n'a pas d'effet sur les performances. À chaque recensement, un enregistrement était effectué de toutes les feuilles mortes : une feuille a été arbitrairement considérée comme morte lorsque plus de $75 \%$ de sa structure avait dépéri.

\section{Mesures destructives}

Quatre prélèvements ont été effectués (expériences 1 et 3 ); les données recueillies à chaque prélèvement concernent hauteur, nombre de tiges par individu, nombre de feuilles total et biomasse par plante.

\section{Fertilisation}

Pour les expériences 1 et 3 , une fumure minérale à base d'engrais simples a été ajoutée, équivalant à 100 unités de $\mathrm{P}_{2} \mathrm{O}_{5}, 100$ unités de $\mathrm{K}_{2} \mathrm{O}$ et 60 unités d'azote/ha.

\section{Analyse statistique}

Les résultats obtenus ont fait l'objet d'une analyse de variance correspondant aux différents dispositifs adoptés et les moyennes ont été classées selon la méthode de Newman et Keuls.

\section{RÉSULTATS}

\section{Rythme d'apparition des feuilles}

\section{Avoine}

\section{Effet de la densité et du mode de culture}

L'intervalle de temps qui sépare l'émergence de 2 feuilles successives est appelé phyllochrone $(\mathrm{PH})$. Les résultats (fig $1 \mathrm{a}, \mathrm{b}$ ) montrent que le rythme d'apparition des feuilles sur la tige principale est variable selon l'ordre du phyllochrone. Le phyllochrone $(\mathrm{PH})$ augmente d'abord, puis accuse une légère diminution pour les $\mathrm{PH}_{3}$ (intervalle de temps compris entre l'apparition de la feuille 2 et celle de la feuille 3 ) et $\mathrm{PH}_{4}$. À partir du $\mathrm{PH}_{5}$, le phyllochrone augmente à nouveau. Sa valeur a varié (densités confondues) pour l'avoine en culture pure (fig 1a) de 64,8 pour $\mathrm{PH}_{5}$ à 153,2 degrés-jours $\left({ }^{\circ} \mathrm{C}\right.$.j) pour $\mathrm{PH}_{9}$, alors que pour l'avoine associée (densités confondues), pour les mêmes phyllochrones, les valeurs ont varié de 59,8 à $131,0^{\circ} \mathrm{C}$.j. L'analyse de variance indique que les effets densité, mode de culture et ordre du phyllochrone sont très hautement significatifs, de même pour les interactions, notamment l'interaction densité $\mathrm{x}$ ordre (fig 1b). Plus la densité augmente, plus le phyllochrone prend des valeurs élevées. Le phyllochrone de la feuille $9\left(\mathrm{PH}_{9}\right)$ a varié pour l'avoine de 107,3 à $179,8^{\circ} \mathrm{C}$.j respectivement pour $d_{1}$ ( $8 \mathrm{ind} /$ pot) et $d_{4}$ (40 ind/pot).

Le phyllochrone est sous la dépendance de la date d'apparition de la feuille et des facteurs trophiques. Sur les talles primaires, le phyllochrone est surtout variable selon l'ordre de la feuille et la densité. Les effets du mode de culture sont marqués; le phyllochrone diminue en association, mais cet effet ne se manifeste qu'à partir du $\mathrm{PH}_{7}$. Pour les talles secondaires, il y a également une interaction phyllochrone $x$ densité et un effet du mode de culture, non illustré ici.

\section{Effet du régime hydrique sur le rythme d'apparition des feuilles}

Le régime hydrique a remarquablement affecté le rythme d'émergence foliaire. Le phyllochrone est d'autant plus long que l'offre en eau du milieu est faible (tous traitements confondus : 127 , 142 et $195^{\circ} \mathrm{C}$.j respectivement pour $\mathrm{H}_{1}, \mathrm{H}_{2}$ et $\mathrm{H}_{3}$ ) et que le rang de la feuille est élevé. En as- 

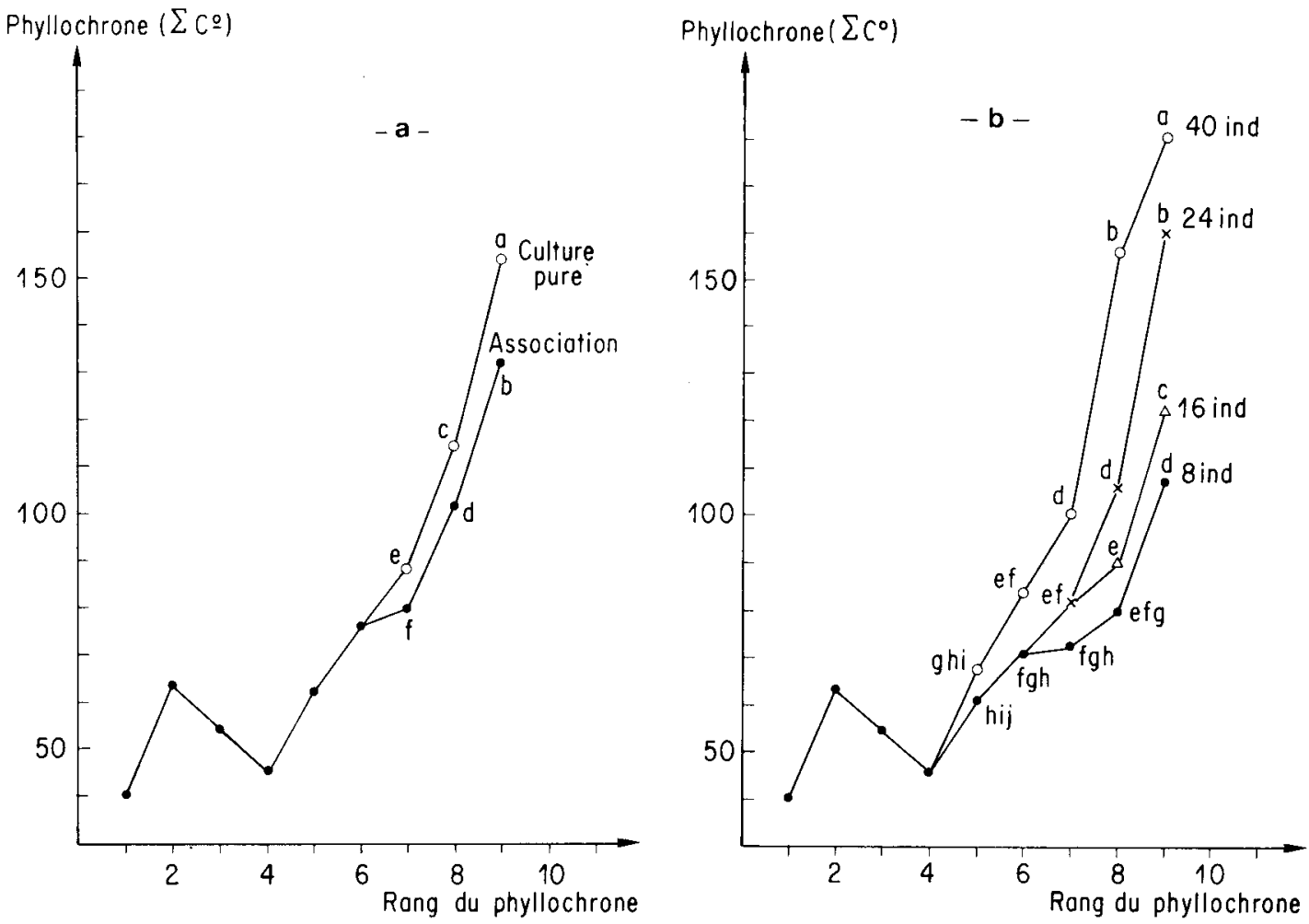

Fig 1. Vitesse d'apparition des feuilles sur le brin maître (tige principale) de l'avoine, selon le rang d'ordre de la feuille, en culture pure et en association (a) et en fonction de la densité (b) (phyllochrone en somme de températures); a $O$ : culture pure, $\bullet$ : association; b : $d_{1}\left(8\right.$ ind/pot); $\Delta d_{2}$ (16 ind/pot); $x: d_{3}$ (24 ind/pot); $O: d_{4}(40$ ind/pot). (Pour toutes les figures, les points remarquables, signalés par des lettres différentes, appartiennent à des groupes de moyennes différents; test de Newman-Keuls au risque 0,05).

sociation, les durées d'émergences sont plus courtes qu'en culture pure. Ainsi, au $\mathrm{PH}_{4}$, les durées en culture pure ont été de 121,2, 140,6, 141,6 et $166,0^{\circ} \mathrm{C}$.j respectivement pour $d_{1}, d_{2}$, $d_{3}$ et $d_{4}$, et, en association, ces durées sont de $105,0,100,6,120,0$ et $128,0^{\circ} \mathrm{C}$.j. Pour un mode de culture donné, l'effet du régime hydrique est plus déterminant que l'effet densité.

\section{Effet du potassium et de l'azote sur le rythme d'apparition des feuilles}

Les résultats obtenus en milieu hydroponique montrent que la déficience en potassium se traduit globalement par une augmentation du phyllochrone qui passe de $100^{\circ} \mathrm{C}$.j pour la concentration 0,6 à 138 pour 0,1 ; le test de NewmanKeuls au seuil $5 \%$ est significatif entre toutes les concentrations (fig 2a). L'apparition des feuilles, exprimée en phyllochrone, est, en fait, en forte interaction avec le rang de ce dernier, le niveau d'azote et celui de potassium (fig 2a) : cette interaction se manifeste essentiellement pour les derniers phyllochrones (rangs $5,6,7$ et 8 , ce dernier non inclus dans l'analyse de variance), et notamment quand il y a un fort déséquilibre entre l'offre en potassium $(0,1)$ et l'offre en azote $(1,4)$. II y a alors un ralentissement important dans l'émission des dernières feuilles. À $0,1 \mathrm{mN}$ de $\mathrm{K}$ la concentration en azote tend à avoir un effet positif sur le phyllochrone.

En culture pure, à $0,3 \mathrm{mN}$ de nitrate, les phyllochrones de l'avoine ont été de 107, 127 et $139^{\circ} \mathrm{C}$.j respectivement pour $0,6,0,3$ et $0,1 \mathrm{mN}$ de $\mathrm{K}$ (tous ordres confondus). Le mode de culture a un effet très significatif (pure : 120; associée : 116). Des phyllochrones nettement plus courts sont enregistrés, en présence de la vesce $: 103,118$ et 131 respectivement pour $0,6,0,3$ et $0,1 \mathrm{mN}$ de $\mathrm{K}$. Le phyllochrone de l'avoine est d'autant plus réduit que la concentration du milieu en nitrate est élevée et ceci quel que soit le mode de culture pour les phyllocrones antérieurs au cinquième phyllochrone.

\section{Vesce}

\section{Effet de la densité}

Comme pour l'avoine, les résultats montrent que le phyllochrone est variable selon la densité et le mode de culture. La contrainte exercée par la 

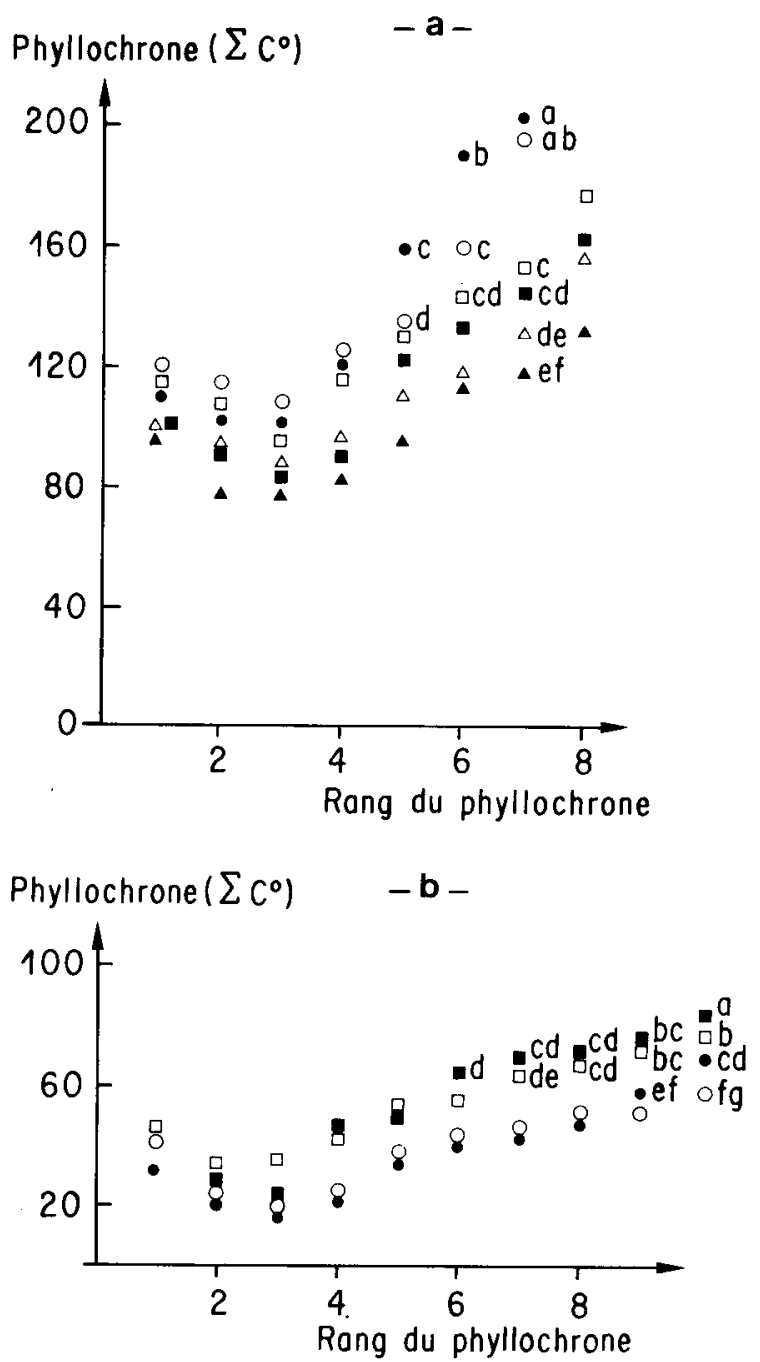

Fig 2. Relations entre le rang du phyllochrone et le phyllochrone en somme de températures. a interaction entre le rang du phyllochrone, le niveau de potassium et le niveau d'azote chez l'avoine. $K^{+} O: 0,1 ;: 0,3 ; \Delta: 0,6 ; 0: 0,1 ; \mathbf{\square}$ : 0,$3 ; \boldsymbol{\Lambda}: 0,6 . \mathrm{NO}_{3}-\mathrm{O}: 0,3 ; \square: 0,3 ; \Delta: 0,3 ; \bullet: 1,4 ; \boldsymbol{\square}: 1,4 ; \boldsymbol{\Lambda}:$ 1,4 . $b$ interaction entre le rang du phyllochrone, le type de culture et le niveau d'azote chez la vesce. Culture $\mathbf{\square}$ : Associée; : Associée; : Pure; $\mathrm{O}:$ pure. $\mathrm{NO}_{3}{ }^{-\mathbf{Q}}: 1,4 ; \mathrm{:}: 0,3 ;$ $: 1,4 ; 0: 0,3$.

densité de peuplement se traduit par des phyllochrones longs (fig $2 b$ ). En association, contrairement à l'avoine, les feuilles émergent plus tardivement qu'en culture pure; la contrainte engendrée par le partenaire avoine s'accentue avec l'ordre de la feuille. À la densité $\mathrm{d}_{4}$, on note, pour le phyllochrone 2 , un décalage de $2,5^{\circ} \mathrm{C} / \mathrm{j}$ et au phyllochrone 6 , de $11^{\circ} \mathrm{C} / \mathrm{j}$ pour la vesce associée.

\section{Effet du régime hydrique} sur le rythme d'apparition des feuilles

La figure 3 met en évidence un effet très marqué, hautement significatif, dans tous les cas, du ré- gime hydrique. Ainsi les valeurs moyennes du phyllochrone (pour l'intervalle $\mathrm{PH}_{2}$ à $\mathrm{PH}_{7}$ ) sont de $89,5,109,0$ et $125,0^{\circ} \mathrm{C}$.j respectivement pour $\mathrm{H}_{1}, \mathrm{H}_{2}$ et $\mathrm{H}_{3}$, conditions de culture confondues.

\section{Effet du potassium et de l'azote sur le rythme d'apparition des feuilles}

Contrairement au cas de l'avoine, c'est surtout le potassium qui est déterminant quant à l'émergence foliaire. À mesure que l'offre en potassium diminue, l'émergence des feuilles est de plus en plus lente. II existe une interaction notable rang du phyllochrone $x$ conditions de culture $x$ azote (fig $2 b$ ), mais le niveau d'azote apporté n'a pas d'effet significatif par lui-même (bien que la croissance ait lieu en conditions d'hydroponie, la fixation symbiotique est probablement active); par contre, l'effet positif du potassium dépend de la dose d'azote (interaction $\mathrm{N} \times \mathrm{K}$ très significative). L'association, quel que soit le niveau d'azote et de potassium, se traduit

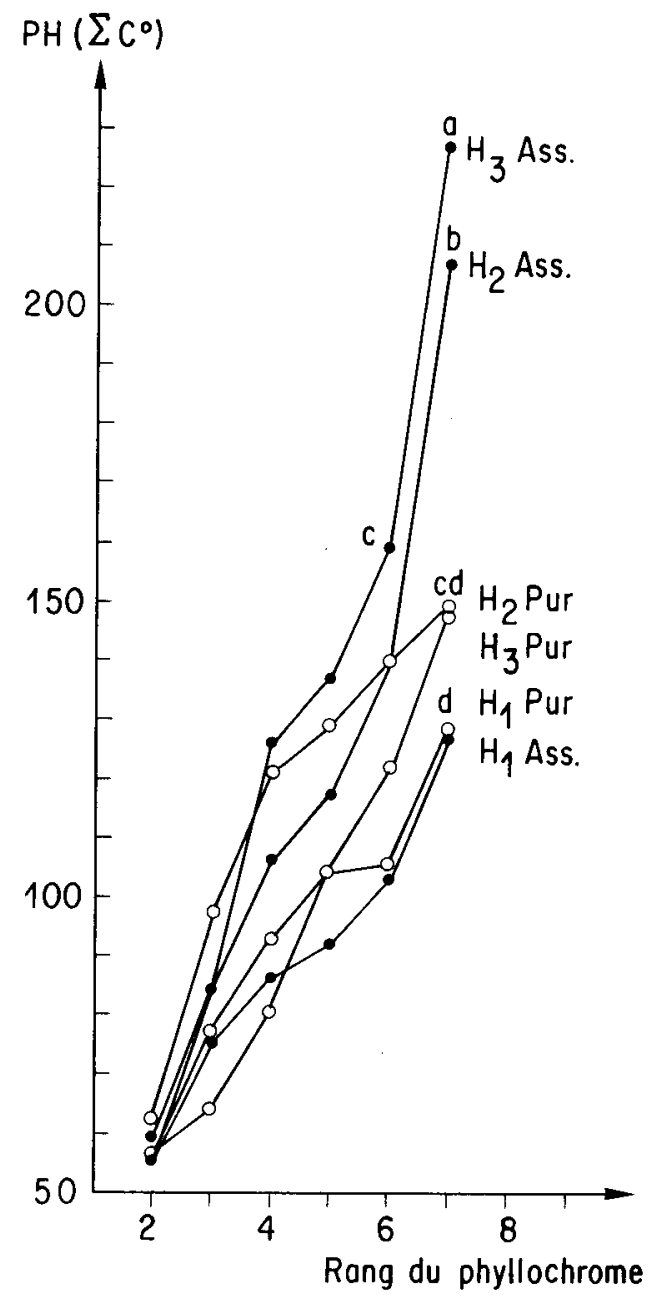

Fig 3. Effet du régime hydrique $(H)$ sur le rythme d'apparition des feuilles $(\mathrm{PH})$ de la tige principale de la vesce en culture pure et en association (Ass) (phyllochrone en somme de températures). 
par des valeurs élevées du phyllochrone moyen (pour l'intervalle $\mathrm{PH}_{1}$ à $\mathrm{PH}_{10}$ ); ce dernier passe de $39^{\circ} \mathrm{C}$.j pour la culture pure à $55^{\circ} \mathrm{C}$.j pour la vesce en association.

\section{Effet de la densité et du mode de culture sur le tallage et la ramification des 2 espèces}

Seul l'effet de la densité et du mode de culture a été abordé, à l'exclusion du rôle de l'azote et du potassium.

\section{Avoine}

Le maximum de tallage est atteint au début du stade montaison pour les densités $d_{2}$ (16 ind/ pot), $d_{3}$ (24 ind/pot) et $d_{4}$ (40 ind/pot), alors qu'à la plus faible densité, $d_{1}$ ( 8 ind/pot), le tallage continue jusqu'au stade pleine montaison. En ce qui concerne le rythme d'apparition des talles, chez l'avoine, l'intervalle de temps nécessaire à l'apparition de la talle de la première feuille, correspondant au début du tallage, n'est affecté, ni par la densité (fig 4a), ni par le mode de culture (résultat non présenté). À densité numérique égale, le tallage par plante est amélioré par la présence de la vesce, d'autant plus que la densité est faible. Pour ce qui a trait à la somme des températures nécessaire à la réalisation d'une talle, les effets de densité et de mode de culture sont très hautement significatifs. La durée d'apparition augmente avec l'ordre de la talle à partir de la talle $T_{2}$. Les interactions : densité $\mathrm{x}$ ordre de la talle et mode de culture $x$ ordre de la talle sont significatives au seuil de $1 \%$. À faible densité $\left(d_{1}\right)$ en culture pure, la durée passe de $48^{\circ} \mathrm{C}$.j pour $T_{2}$ à $90^{\circ} \mathrm{C}$.j pour $\mathrm{T}_{4}$. À forte densité $\left(\mathrm{d}_{4}\right)$, la durée est passée de 51 à $127^{\circ} \mathrm{C}$.j. Les talles d'ordres supérieurs : $T_{1 i}, T_{2 k}, T_{3 j}, T_{41}$ (fig 4b) ne présentent pas la même tendance à l'augmentation de la durée avec l'ordre des talles, mais l'effet de la densité est net (sauf pour $T_{1 i}$ ).

\section{Vesce}

Contrairement à l'avoine, lạ ramification de la vesce a commencé très tôt, dès la levée; toutefois, un intervalle de temps entre l'apparition des rameaux sur la tige (initiation) et leur départ en végétation (première feuille) semble être nécessaire (tableau I). Ce temps de latence est sous l'influence de la température; en effet, exprimé en jours, le temps nécessaire au départ en végé-

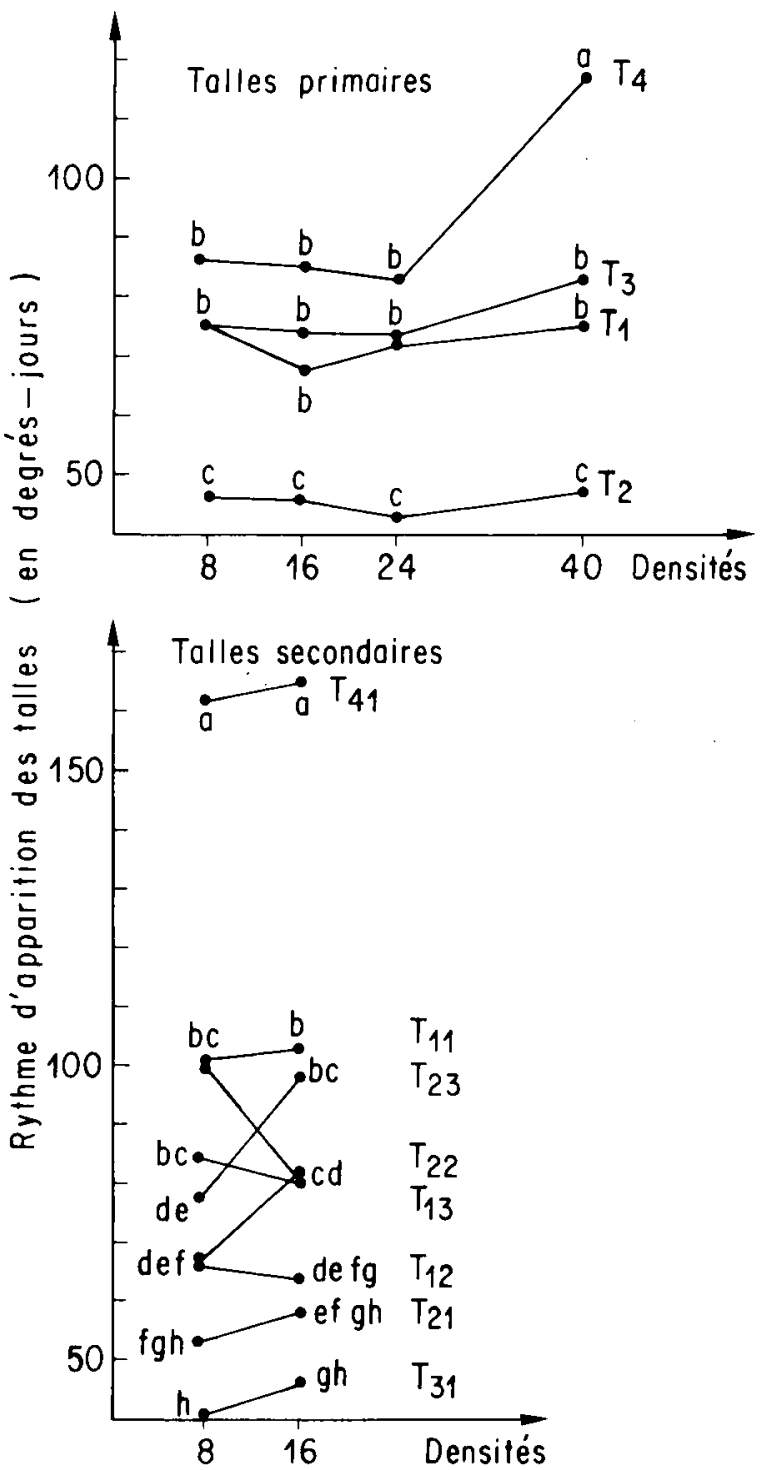

Fig 4. Effet de la densité $(D)$ sur le rythme d'apparition des talles chez l'avoine (Durée exprimée en ${ }^{\circ} \mathrm{C} . j$ ). $T_{j}$ : l'indice indique l'ordre de la feuille; $T_{i j}$ : le premier chiffre indique l'ordre de la talle primaire et le second l'ordre de la feuille dont elle est issue.

tation diminue avec l'ordre des rameaux à partir de $R_{3}$ (tableau $I A$ ) : les intervalles les plus longs correspondent à des températures moyennes journalières faibles et inversement. Ce type de réponse ne semble pas se confirmer lorsqu'on intègre la température, comme c'est le cas lorsque l'on utilise les degrés-jours (tableau IB). Ceci pourrait s'expliquer par l'existence d'une constante de ramification liée à l'acquisition d'une quantité donnée d'énergie. L'importance de la ramification (apparition de rameaux d'ordre 5 et 6 ) est sous l'influence de la densité de peuplement et du mode de culture (tableau I). L'analyse de 
Tableau I. Intervalle de temps entre l'apparition d'un rameau et son départ en végétation chez la vesce. A : temps en jours; $\mathrm{B}$ : temps en somme de temperatures.

\begin{tabular}{|c|c|c|c|c|c|c|c|c|c|c|c|c|c|}
\hline & & \multicolumn{2}{|c|}{$R_{1}$} & \multicolumn{2}{|c|}{$R_{2}$} & \multicolumn{2}{|c|}{$R_{3}$} & \multicolumn{2}{|c|}{$R_{4}$} & \multicolumn{2}{|c|}{$R_{5}$} & \multicolumn{2}{|c|}{$R_{6}$} \\
\hline & & $A$ & $B$ & $A$ & $B$ & $A$ & $B$ & $A$ & $B$ & $A$ & $B$ & $A$ & $B$ \\
\hline$D_{1}$ & $\begin{array}{l}V_{p} \\
V_{a}\end{array}$ & $\begin{array}{l}11,5 \\
13,5\end{array}$ & $\begin{array}{l}40,6 \\
47,25\end{array}$ & $\begin{array}{l}16 \\
18,0\end{array}$ & $\begin{array}{l}53,6 \\
63,54\end{array}$ & $\begin{array}{l}6,5 \\
6,0\end{array}$ & $\begin{array}{l}54 \\
50,5\end{array}$ & $\begin{array}{l}6,0 \\
6,5\end{array}$ & $\begin{array}{l}41,6 \\
54,14\end{array}$ & $\begin{array}{l}4,33 \\
5\end{array}$ & $\begin{array}{l}52,7 \\
41,6\end{array}$ & $\begin{array}{l}3,5 \\
\text { ND }\end{array}$ & $\begin{array}{l}29,0 \\
-\end{array}$ \\
\hline $\mathrm{D}_{2}$ & $\begin{array}{l}V_{p} \\
V_{a}\end{array}$ & $\begin{array}{l}14 \\
13,5\end{array}$ & $\begin{array}{l}49,4 \\
47,25\end{array}$ & $\begin{array}{l}15,8 \\
16\end{array}$ & $\begin{array}{l}55,99 \\
56,48\end{array}$ & $\begin{array}{l}7,0 \\
6,8\end{array}$ & $\begin{array}{l}58,0 \\
66,6\end{array}$ & $\begin{array}{l}7,33 \\
6,3\end{array}$ & $\begin{array}{l}45,0 \\
58,5\end{array}$ & $\begin{array}{l}7,0 \\
6,8\end{array}$ & $\begin{array}{l}62,47 \\
56,64\end{array}$ & $\begin{array}{l}4 \\
3,75\end{array}$ & $\begin{array}{l}33,3 \\
25,0\end{array}$ \\
\hline$D_{3}$ & $\begin{array}{l}V_{p} \\
V_{a}\end{array}$ & $\begin{array}{l}12,85 \\
14,8\end{array}$ & $\begin{array}{l}45,36 \\
52,24\end{array}$ & $\begin{array}{l}15 \\
14,9\end{array}$ & $\begin{array}{l}56,5 \\
52,6\end{array}$ & $\begin{array}{l}6,1 \\
5,5\end{array}$ & $\begin{array}{l}50,8 \\
45,81\end{array}$ & $\begin{array}{l}6,40 \\
6,25\end{array}$ & $\begin{array}{l}53,35 \\
59,25\end{array}$ & $\begin{array}{l}4,78 \\
5,5\end{array}$ & $\begin{array}{l}39,81 \\
45,8\end{array}$ & $\overline{N D}$ & $\begin{array}{c}36,0 \\
-\end{array}$ \\
\hline $\mathrm{D}_{4}$ & $\begin{array}{l}V_{p} \\
V_{a}\end{array}$ & $\begin{array}{l}15,65 \\
14,9\end{array}$ & $\begin{array}{l}55,24 \\
52,6\end{array}$ & $\begin{array}{l}18,0 \\
16,6\end{array}$ & $\begin{array}{l}63,5 \\
58,31\end{array}$ & $\begin{array}{l}7,8 \\
7,5\end{array}$ & $\begin{array}{l}52,0 \\
58,31\end{array}$ & $\begin{array}{l}7,2 \\
8,85\end{array}$ & $\begin{array}{l}68,3 \\
79,0\end{array}$ & $\begin{array}{l}6,33 \\
\text { ND }\end{array}$ & $\begin{array}{c}37,5 \\
-\end{array}$ & $\begin{array}{l}5,5 \\
\text { ND }\end{array}$ & $\begin{array}{l}- \\
-\end{array}$ \\
\hline
\end{tabular}

$R$ : rameau. $D$ : densité; $V_{p}$ : vesce pure; $V_{a}$ : vesce associée; ND : non développé.

variance du temps en ${ }^{\circ} \mathrm{C} . \mathrm{j}$ montre que le départ en végétation dépend des modalités de culture : il est significativement retardé par l'association. En outre, à forte densité, ce retard est très accentué pour $R_{4}$.

\section{La durée de vie des feuilles}

\section{Avoine}

\section{Effet de la densité et du régime hydrique}

Les mesures ont été faites uniquement au cours de l'expérience 3 , et concernent les seules feuilles de la tige principale. Les résultats montrent que la densité et le régime hydrique ont affecté différemment la longévité des feuilles. L'augmentation de la densité se traduit par les durées de vie plus courtes si l'offre en eau est optimale $\left(H_{1}\right): 574^{\circ} \mathrm{C}$.j à $d_{1}$ contre 550 à $d_{4}$. Par contre, la réduction de l'offre en eau augmente la longévité des feuilles (fig 5a) : les feuilles sont produites plus lentement et tendent à vivre plus longtemps. La présence de la vesce se traduit aussi par une augmentation de la longévité des feuilles.

\section{Vesce}

\section{Effet de la densité et du régime hydrique}

En culture pure et pour le régime $\mathrm{H}_{1}$ (offre en eau non limitante), les durées de vie des feuilles (tous ordres confondus) sont de 502,6; 471,6;
429,2 et $415,8^{\circ} \mathrm{C}$.j respectivement pour $d_{1}, d_{2}$, $d_{3}$ et $d_{4}$. L'augmentation de la densité de peuplement avance la mortalité des feuilles, quel que soit le régime hydrique considéré et le mode de culture (fig 6). Les feuilles ne sont pas seulement produites plus lentement mais tendent à avoir des durées de vie plus courtes. Par contre, un régime hydrique déficient retarde la mortalité foliaire (fig $5 \mathrm{~b}$ ). Ainsi, la durée de vie pour le régime $\mathrm{H}_{3}$ est de $665,4,651,6,630,4$ et $609,4^{\circ} \mathrm{C}$.j respectivement pour $d_{1}, d_{2}, d_{3}$ et $d_{4}$ (tous ordres de feuilles confondus). L'association avance la mortalité des feuilles.

\section{Effet de la déficience potassique}

La figure 7 met en évidence un effet très net de l'offre en potassium sur la durée de vie des feuilles. Cette durée de vie pour un mode de culture donné diminue avec la diminution de la disponibilité en potassium du milieu, effet d'autant plus marqué que le niveau d'azote est élevé. Modes de culture et feuilles confondus, à 0,3 $\mathrm{mN}$ de nitrate, les durées de vie sont de 593,3 , 517,0 et $412,0^{\circ} \mathrm{C}$.j respectivement pour $0,6,0,3$ et $0,1 \mathrm{mN}$ de $\mathrm{K}$. De même, à $1,4 \mathrm{mN}$ de nitrate, les durées de vie sont de 564,8, 405,9 et $317,0^{\circ} \mathrm{C}$.j respectivement. Le niveau d'azote réduit la durée de vie des feuilles. L'effet partenaire avoine est similaire à celui de l'azote : en effet, à $0,3 \mathrm{mN}$ de nitrate (tous ordres de feuille confondus), la durée de vie en culture pure est. de $616,7,587,0$ et $454,6^{\circ} \mathrm{C}$.j et en association $568,8,447,0$ et $369,4^{\circ} \mathrm{C}$.j respectivement pour $0,6,0,3$ et $0,1 \mathrm{mN}$ de $\mathrm{K}$. 

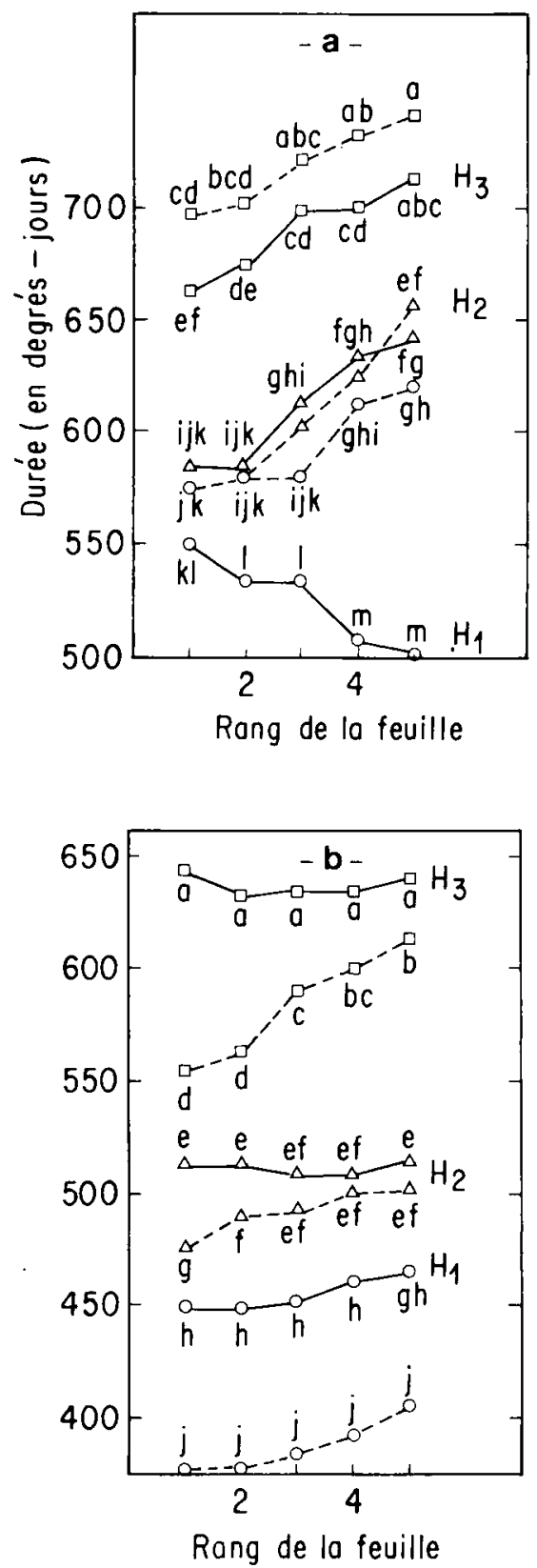

Fig 5. Effet du régime hydrique $(H)$ sur la durée de vie (en $\left.{ }^{\circ} \mathrm{C} . j\right)$ des feuilles de l'avoine (a) et de la vesce (b) en culture pure (- $\longrightarrow$ ) et en association (- - - ) .

\section{DISCUSSION ET CONCLUSION}

Gounot et al (1980) ont montré l'existence d'une relation linéaire entre le logarithme de la croissance en biomasse et le phyllochrone. Dans les limites où cette relation est valable, elle implique le déclenchement d'un phyllochrone chaque fois que la biomasse est multipliée par un facteur constant. Billiard et Gounot (1989) ont présenté récemment un modèle descriptif de la cinétique d'allongement des feuilles qui souligne l'intégra-

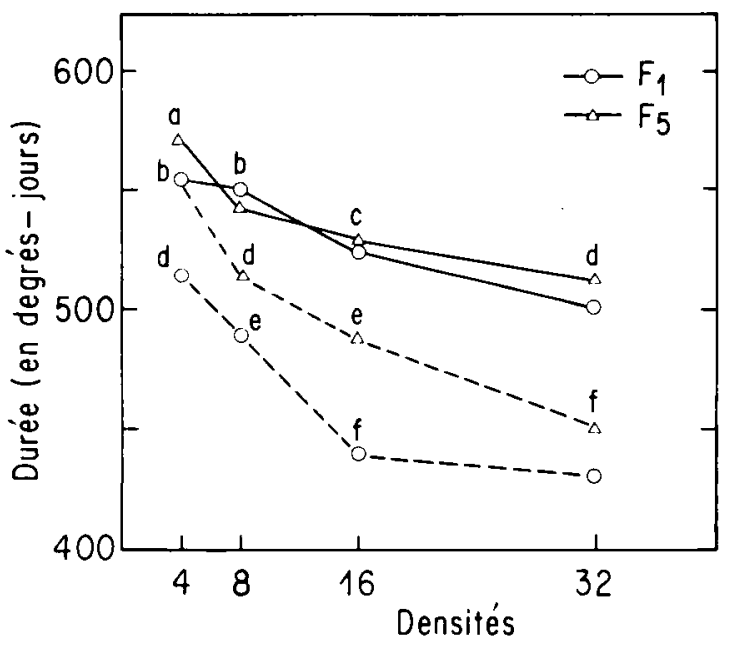

Fig 6. Effet de la densité sur la durée de vie (en $\left.d^{\circ} \mathrm{C} . j\right)$ des feuilles $\left(F_{1}\right.$ et $\left.F_{5}\right)$ de la vesce en culture pure (et en association $(----)$.

tion du fonctionnement au niveau de la talle et l'intérêt d'une approche phyllochronique de la croissance. Selon Lemaire et al (1988), il semble que la morphogenèse soit plus sensible aux contraintes hydriques et à la carence en azote que la photosynthèse. Les facteurs limitants joueraient sur la répartition des produits de cette dernière, en favorisant la mise en réserve, plutôt que la formation de nouveaux organes.

Les résultats présentés, qui recoupent un grand nombre de travaux réalisés dans ce domaine (Bazzaz et Harper, 1977; Maitre, 1981; Turkington, 1983; Maitre et al, 1985), font ressortir que lorsque les plantes interfèrent en association comme en culture pure (effet densité), la concurrence agit de façon différente sur le rythme d'émergence foliaire selon l'espèce (vesce ou avoine) et la nature de la concurrence. Sur la vesce, les concurrences, intra- et interspécifiques, agissent de la même façon en ralentissant la vitesse d'apparition des feuilles. Le phyllochrone, indice retardé du plastochrone, a une durée qui est d'autant plus grande que le rang de la feuille est élevé. Pour une feuille d'ordre donné, il est d'autant plus long que la plante est soumise à une contrainte. Dans nos expériences, le phyllochrone présente une importante variabilité apparente qui reste encore en grande partie à expliquer. On peut se demander si le phyilochrone n'est pas une fonction du rang d'une feuille sur la tige et/ou de l'ordre de tallage et de la date d'apparition de la feuille. Sur l'avoine, la concurrence interspécifique se traduit 

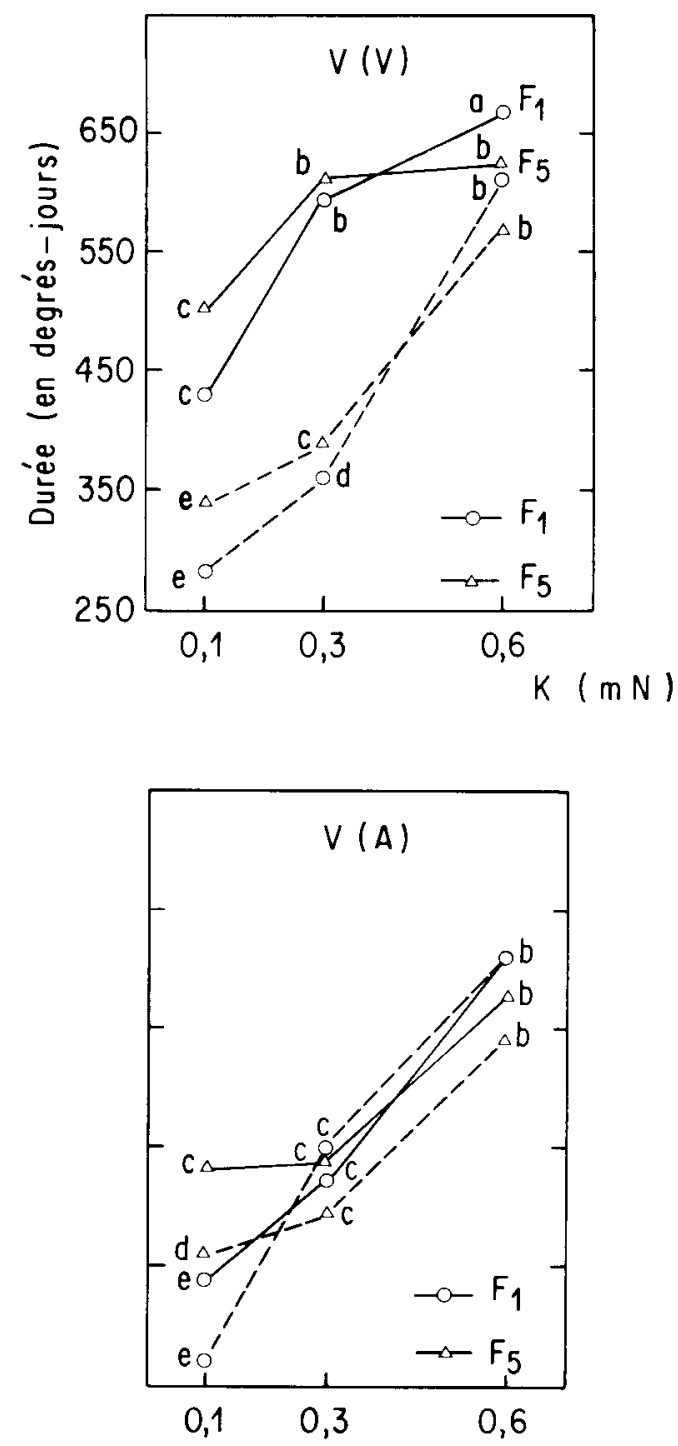

Fig 7. Effet de la déficience potassique $(K)$ et du niveau d'azote (— : $0,3 \mathrm{mN} \mathrm{NO}_{3}^{-} ;----: 1,4 \mathrm{mN} \mathrm{NO}_{3}{ }^{-}$) sur la durée de vie (en ${ }^{\circ} \mathrm{C}$.j) des feuilles de la vesce $\left(F_{1}\right.$ et $\left.F_{5}\right)$ en culture pure, $V(V)$, et en association, $V(A)$.

par une production de feuilles élevée qui s'explique par des phyllochrones raccourcis. Parmi les contraintes étudiées, l'effet du régime hydrique est beaucoup plus déterminant que l'effet densité. En culture pure, l'avoine se montre beaucoup plus sensible au déficit hydrique que la vesce. Ainsi, le passage de $\mathrm{H}_{1}$ à $\mathrm{H}_{3}$ (offre réduite de $75 \%$ par rapport au témoin $\mathrm{H}_{1}$ ) a entraîné une augmentation du phyllochrone de 53,5\% sur l'avoine et seulement $40 \%$ sur la vesce. Par contre, la densité n'a entraîné qu'une augmentation de $37 \%$ du phyllochrone sur l'avoine et $26 \%$ seulement sur la vesce. La déficience en potassium s'est traduite sur les 2 espèces par une augmentation du phyllochrone de l'ordre de $20 \%$.
Des résultats obtenus par Balfourier (non publiés) concernant le potentiel de croissance de la luzerne en relation avec le déficit hydrique, et notamment les aspects morphologiques, montrent que la sécheresse se traduit par un arrêt de l'émission de nouveaux entre-nœuds et un ralentissement de l'élongation cellulaire conduisant à des tiges plus courtes qu'en l'absence de déficit et portant plus de feuilles. Une contrainte hydrique, par contre, chez les 2 espèces étudiées ici, se traduit par un rythme de production de feuille ralenti, associé à une longévité élevée. Ceci entraîne au niveau de la plante une réduction de l'appareil évaporant aboutissant à établir un équilibre entre la quantité d'eau évapotranspirée et la quantité d'eau disponible (susceptible d'être absorbée par les racines).

Bazzaz et Harper (1977) ont signalé qu'une déficience en éléments nutritifs augmentait la vitesse de mortalité des feuilles et que l'application foliaire d'un fertilisant combinant l'azote et le potassium réduisait la mortalité et compensait partiellement le déficit minéral. Chapin (1980) a rapporté des résultats de même nature et que la sénescence est une réponse commune des plantes à une offre inadéquate du milieu en nutriments. D'autres auteurs ont signalé que la mortalité des feuilles commence quand la biomasse du peuplement atteint le recouvrement total (par rapport à la surface du sol qu'il occupe) (Bazzaz et Harper, 1977). La mortalité agit alors comme un régulateur de l'indice foliaire (Maitre, 1981). Nos résultats montrent que sous l'effet de l'augmentation de la densité et de la déficience en potassium les feuilles ne sont pas seulement produites plus lentement mais leur longévité est réduite.

Le tallage, chez l'avoine, se déroule selon la séquence décrite au champ par Masle-Meynard (1980) : la talle de rang 1 devient visible audessus de la ligule de la première feuille lorsque le brin-maître possède 4 feuilles. Les talles successives sont émises au même rythme que les feuilles jusqu'à l'apparition de la sixième feuille, stade où le synchronisme est interrompu. Le temps de latence de la ramification chez la vesce dépend non seulement de la température mais aussi, semble-t-il, de la position du rameau sur la tige principale et/ou de facteurs hormonaux; sur Salix babylonica L, Letouze (1972) a mis en évidence un parallélisme entre la teneur en phytochrome actif et la croissance du bourgeon axillaire.

L'organisation morphologique des 2 espèces étudiées est donc très sensible aux contraintes 
imposées par les variables de ressource et par les caractéristiques du peuplement (pur ou associé).

\section{RÉFÉRENCES}

Bazzaz FA, Harper JL (1977) Demographic analysis of the growth of Linum usitatissimum. New Phytol 78, 193-208

Billiard D, Gounot M (1989) Modèle de la cinétique d'allongement des feuilles successives sur une talle d'Hyparrhenia diplandra (Hochst). CR Séances Acad Sci (Paris) 309, III, 675-681

Chapin FS (1980) The mineral nutrition of wild plants. Annu Rev Ecol Syst 11, 233-260

Donald CM (1963) Competition among crop and pasture plants. Adv Agron 15, 1-118

Fowler N, Antonovics J (1981) Small-scale variability in the demography of transplants of two herbaceous species. Ecology 62, 1450-1457

Gounot M, Atry M, N'kandza J, Yu O (1980) Photosynthèse nette et rythme d'apparition des feuilles chez le dactyle (Dactylis glomerata L). CR Séances Acad Sci 290, Ser D, 1257-1260

Harper JL (1961) Approaches to the study of plant competition. In: Mechanisms in biological competition. (FL Milhorpe, ed) vol 15, 1-39

Jacquard $P$ (1984) Influence de la densité sur la morphogenèse et la production de Bromus erectus Huds. Oecol Plant, vol 5(19) n ${ }^{\circ}$ 1, 15-37

Lemaire G, Varlet-Granchet C, Gastal F, Durand JL (1988) Ecophysiological approach of plant growth. Consequences on breeding strategies of forage species for contrasted conditions and different managements, in: Natural variation and breeding for adaptation. Proc Eucarpia Fodder Crop Sect Meeting, INRA, Lusignan, 103-118
Letouze MR (1972) Phytochrome et croissance du bourgeon axillaire chez Salix babylonica L. CR Séances Acad Sci (Paris) T, 275, Ser D, 2663-2666

Mack R, Harper JL (1977) Interference in dune animals: Spatial pattern and neighbourhood effects. $J$ Ecology 65, 345-363

Maitre JP (1981) Dynamique des populations d'organes et de la production de matière sèche chez Trifolium pratense $\mathrm{L}$ : rôle de la concurrence. Thèse Doc Ing, USTL, Montpellier, $122 \mathrm{p}$

Maitre JP, Assemat L, Jacquard P (1985) Croissance du trèfle violet (Trifolium pratense $L$ ) en association avec du ray grass d'Italie (Lolium multiflorum Lam ssp ltalicum). I. Description de l'organisation morphologique du trèfle; II. Démographie des organes, biomasse et interférences biologiques. Agronomie $5,251-260$ et $297-304$

Masle-Meynard J (1980) L'élaboration du nombre d'épis chez le blé d'hiver. Influences de différentes caractéristiques de la structure du peuplement sur l'utilisation de l'azote et de la lumière. Thèse Doc ing, INA-PG, Paris

Obeid M, Machin D, Harper JL (1967) Influence of density on plant to plant variations in fiber flax, Linum usitatissimum. Crop Sci 7, 471-473

Ogden J (1970) Plant population structure and productivity. Proc NZ Ecol Soc 17, 1-9

Ouknider M, Jacquard $P$ (1986) Production et valeur nutritive de l'association vesce-avoine en zone méditerranéenne. Fourrages 105, 39-62

Ouknider M, Jacquard P (1988) Un modèle d'association graminée-légumineuse : Le mélange Vesce (Vicia sativa L) - avoine (Avena sativa L). Agronomie 8, 97-106

Turkington R (1983) Leaf and flower demography of Trifolium repens L. I. Growth in mixture with grasses. New Phytol 93, 599-616 